\title{
Chitosan-Glutathione Nanoparticles Modify Hepatic Cellular Response on Bovine Precision-cut Liver Slices Treated With Zilpaterol and Clenbuterol.
}

\section{Sofia Piña Olmos}

Universidad Nacional Autonoma de Mexico

Mariana Dolores Hernández

Universidad Nacional Autonoma de Mexico

Roberto Díaz Torres

Universidad Nacional Autonoma de Mexico

\section{J. Efrén Ramírez Bribiesca}

Colegio de Postgraduados Campus Montecillo: Colegio de Postgraduados

Raquel López Arellano

Universidad Nacional Autonoma de Mexico

Patricia Ramírez Noguera ( $\square$ ramireznoguera@unam.mx )

Universidad Nacional Autónoma de México: Universidad Nacional Autonoma de Mexico https://orcid.org/0000-0002-0900-9162

\section{Research Article}

Keywords: clenbuterol, zilpaterol, chitosan-glutathione nanoparticles, precision-cut liver slices

Posted Date: June 4th, 2021

DOl: https://doi.org/10.21203/rs.3.rs-563479/v1

License: (c) (1) This work is licensed under a Creative Commons Attribution 4.0 International License. Read Full License 


\section{Abstract}

Zilpaterol and clenbuterol are two $\beta$-adrenergic agonist drugs used on animal production. Both drugs have anabolic effects with advantages on carcass yield. Meanwhile, zilpaterol is approved for animal feed in authorized countries; clenbuterol is a banned substance due to the risk of toxicity; however, it is still being used at unknown dose levels in many farm species. Therefore, the use and abuse of these substances should be closely monitored, considering the clenbuterol ability and the not proven yet of zilpaterol to produce reactive oxygen (ROS) and nitrogen species. Regarding glutathione which is the main intracellular antioxidant and plays detoxification functions on liver metabolism; case study is a primary interest to know the capacity of chitosan-glutathione nanoparticles (CS/GSH-NP) as a complementary source of exogenous GSH to modify the oxide-reduction status on bovine precision-cut liver slice cultures exposed to clenbuterol and zilpaterol. A single drug assay was performed in the first instance by adding clenbuterol, zilpaterol, chitosan nanoparticles (CS-NP), and CS/GSH-NP. Then combinate drug assay was carried out by testing clenbuterol and zilpaterol combined with CS-NP or CS/GSH-NP. The results showed that both agonist $\beta$-adrenergic modify in a dose-dependent manner oxide-reduction response through ROS generation, glutathione peroxidase activity, and intracellular GSH content; and de release of liver enzymes associated with hepatocellular damage like gamma glutamyltranspeptidase, aspartate aminotransferase, alanine aminotransferase. The exogenous GSH delivered by nanoparticles could be used to modulate these markers.

\section{Introduction}

Zilpaterol and clenbuterol are two $\beta$-adrenergic agonists used to improve growth performance, feed efficiency, and carcass quality in cattle (Centner et al. 2014; Milford et al. 2019). While zilpaterol is a lawful drug for cattle feed in authorized countries (Choi et al. 2013; Cônsolo et al. 2016), clenbuterol is restricted to therapeutic applications. Both drugs have similar pharmacokinetic and pharmacodynamic (FAO and WHO Expert Committee on Food Additives 2016; FAO and WHO Expert Committee on Food Additives 2018). However, their toxicokinetic and toxicodynamic are not well established, There are also some toxicological concerns on animals and the risk population due to the misuse of these substances on animal feeding. When they are orally administrated in laboratory animals, domestic species, and humans (FAO and WHO Expert Committee on Food Additives 2016); The target organs involved in the metabolism and excretion are the liver and kidneys (FAO and WHO Expert Committee on Food $\ll$ «Additives 2014). Once these $\beta$-adrenergic reach the liver tissue, phases I and II react such as hydroxylation, n-oxidation, conjugation with sulphates and glucuronides occurs (Zalko et al. 1997; Alonen et al. 2009; Domínguez-Romero et al. 2013); some of these arisen substances could be toxic for hepatic cells by the formation of reactive oxygen species (ROS) or reactive nitrogen species (NOS), leading to oxide-reduction unbalance and cellular damage.

Previous reports demonstrate that antioxidants such as ascorbic acid and reduced glutathione (GSH) prevent reactive metabolites formation during clenbuterol metabolism (Brambilla et al. 2007). 
GSH is a low-molecular-weight, non-protein thiol made up of glycine, cysteine, and glutamic acid synthesized and stored in the liver. As well as the main antioxidant of cellular origin that only can be synthesized in the cellular cytoplasm (Lu 2009), to exercise functions like reactive species scavenger, regulating the oxide reduction balance (Patlevič et al. 2016), participate in the xenobiotic detoxification processes by the conjugation with xenobiotic and their metabolites, cell signalling and others biochemical reactions; situations that limit the cellular GSH quantity many times; thus, the cell needs de novo synthesis and trigger the start-up of other enzymatic and non-enzymatic mechanisms to maintain cellular homeostasis (He et al. 2017).

Several research techniques to evaluate drug safety and toxicity effects; in vitro techniques are widely used to assess the effects of xenobiotic substances. Unlike in vivo studies with humans or animals, invitro techniques are versatile tools adaptable to ethical standards, with a short evaluation time, low cost, and high efficacy of the data generated (Ruoß et al. 2020). Particularly, precision-cut liver slice cultures (PCLS) have the advantage of reducing the number of experimental animals (Soldatow et al. 2013; Granitzny et al. 2017); better control in environmental conditions, a reduction in genetic heterogeneity, and due to the limited amount of the drug evaluated, there is less contamination by organic waste (Ruoß et al. 2020); additionally, the three-dimensional structure in the liver tissue is preserved, with good correlation on in vivo studies.

As mentioned above, GSH prevents the formation of reactive metabolites during clenbuterol metabolism; however, there is no information related to zilpaterol metabolism (Lu 2013; Lu 2020), so the use of chitosan nanoparticles offer an alternative to deliver GSH into the cell and counteract the adverse effects caused by GSH depletion; because chitosan is a biocompatible, biodegradable, and low toxicity polymer (Van der Lubben et al. 2001) widely used in thiol nanoparticle designing (Kafedjiiski et al. 2005; Kumar \& Sinha 2013; Hejjaji et al. 2018).

In this study, was utilized bovine PCLS and applied as a tool to evaluate clenbuterol and zilpaterol on hepatic cells through the release of hepatic enzymes and oxide-reduction markers (Masubuchi et al. 2016). Since clenbuterol and zilpaterol are metabolized in the liver, the bovine PCLS are an adequate strategy to explore the biological and toxicological effects of these $\beta$-adrenergic drugs, considering the combination of each one with chitosan-glutathione nanoparticles (CS/GSH-NP). GSH plays an essential role in hepatic metabolism and may reduce the hepatocyte damage caused by clenbuterol and zilpaterol treatment by considering the limited cytoplasmatic synthesis of GSH.

\section{Materials And Methods}

\section{Reagents}

Clenbuterol hydrochloride was purchased from Sigma-Aldrich (St. Louis, MO., USA) and zilpaterol hydrochloride from Santa Cruz Technology (Dallas, TX., USA). Glacial acetic acid, chitosan low molecular weight (CS), sodium tripolyphosphate (TPP), reduced glutathione (GSH), D-Glucose, $\mathrm{MgCl}_{2}, \mathrm{CaCl}_{2}$, 
$\mathrm{NaHCO}_{3}, \mathrm{MgSO}_{4}$, and ammonium acetate were purchased from Sigma-Aldrich (St. Louis, MO., USA); $\mathrm{NaOH}, \mathrm{ZnOH}$, Acetonitrile, $\mathrm{KCl}, \mathrm{NaCl}, \mathrm{KH}_{2} \mathrm{PO}_{4}, \mathrm{Na}_{2} \mathrm{HPO}_{4}$ were from JT. Baker (Hampton, $\mathrm{NH}$., USA) and formic acid were from Fermont (NL., Mex.).

\section{Nanoparticle synthesis and physicochemical characterization}

Chitosan nanoparticles (CS-NP) and glutathione-chitosan nanoparticles (CS/GSH-NP) were prepared by the ionic gelation method (Koo et al. 2011; Piña-Olmos et al. 2019). Briefly, CS $0.3 \%$ and TPP $0.1 \%$ solutions were made using acetic acid $1 \%$ as a solvent. For CS-NP, a CS solution volume was mixed with another volume of TPP and stirred for one hour. CS/GSH-NP were made with $0.5 \%$ of GSH mixed with CS solution and left under magnetic stirring for 60 minutes. Then, TPP was added into the mix and left for another 60 minutes. The nanoparticles formed were filtered through a $0.45 \mu \mathrm{m}$ membrane and kept at $4^{\circ} \mathrm{C}$ until use. Physicochemical characterization was carried by measuring loading efficiency, size, surface charge, and morphology using nanoparticle tracking analyses (Zeta View, Particle Metrix, Ammersee Germany).

\section{Preparation of precision-cut liver slices}

Bovine liver samples were obtained from animals slaughtered in the municipal slaughterhouse of Zumpango, Mexico State, Mexico, following the local care legislation NOM-033-SAG/Z0O-2014. Methods to slaughter domestic and wild animals (Diario Oficial de la Federación 2015). The animals were finished in a feedlot pen, and during the feeding period, they did not receive any type of $\beta$-adrenergic drug. The livers were collected, washed with cold Krebs-Henseleit buffer (D-glucose $11 \mathrm{mM}, \mathrm{MgSO}_{4} 1.2 \mathrm{mM}$, $\mathrm{KH}_{2} \mathrm{PO}_{4} 1.2 \mathrm{mM}, \mathrm{KCl} \mathrm{mM} 4.7 \mathrm{mM}, \mathrm{NaCl} 118 \mathrm{mM}, \mathrm{CaCl}_{2} 1.25 \mathrm{mM}, \mathrm{NaHCO}_{3} 25 \mathrm{mM} \mathrm{pH} 7.4$ ) and transported to the laboratory; in addition, the tissue fractions were placed on a tissue slicer Leica VT 1200 (Leica Biosystems, Wetzlar, Germany) containing dissection solution ( $\mathrm{CaCl}_{2} 1 \mathrm{mM}, \mathrm{D}$-glucose $10 \mathrm{mM}, \mathrm{KCl}$ $4 \mathrm{mM}, \mathrm{MgCl}_{2} 5 \mathrm{mM}, \mathrm{NaHCO}_{3} 26 \mathrm{mM}$, phenol red $0.1 \%, \mathrm{pH}$ 7.4). Then, to be cut with an oscillating blade, where was obtained slices of $200 \mathrm{~mm}-250 \mathrm{~mm}$ wide and $8 \mathrm{~mm}$ in diameter.

\section{Bovine precision-cut liver slices culture}

Precision-cut liver slices (PCLS) were placed on 6-well polystyrene plates containing $3.0 \mathrm{~mL}$ of cell culture medium DMEM (Corning, NY., USA) supplemented with 10\% fetal bovine serum (Sigma-Aldrich, MO., USA) and $1 \%$ of antibiotic-antifungal solution $100 x$ (Gibco, M.A., USA). PCLS were incubated at $37^{\circ} \mathrm{C}$, with $95 \%$ of $\mathrm{O}_{2}, 5 \%$ of $\mathrm{CO}_{2}$ for one hour to normalize culture conditions before starting the treatment (Olinga \& Schuppan 2013).

\section{Single drug and nanoparticles exposure}

Single treatments were carried out on PCLS treated with two concentrations of zilpaterol (10 and 25 $\mathrm{ng} / \mathrm{mL}$ ), clenbuterol (10 and $25 \mathrm{ng} / \mathrm{mL}$ ), CS-NP (10 and $25 \mu \mathrm{g} / \mathrm{mL})$, and CS/GSH-NP (10 and $25 \mu \mathrm{M} \mathrm{GSH})$ 
and left under culture conditions for 6 hours (Fig. 1), For good measure, cultures were harvested and stored at $-80^{\circ} \mathrm{C}$. An unthreaded group culture was considered as the negative control.

\section{Combined drug-nanoparticles exposure}

PCLS cultures were exposed to a factorial arrangement $2 \times 2$. First, the cultures were exposed to 10 and 25 $\mathrm{ng} / \mathrm{mL}$ of zilpaterol and clenbuterol for 3 hours, then each zilpaterol were added two amounts of the nanoparticles (zilpaterol level x CS-NP level; zilpaterol level x CS/GSH-NP level), and clenbuterol (clenbuterol level x CS-NP level; clenbuterol level x CS/GSH-NP level) after while were left under incubation another $3 \mathrm{~h}$ (Fig. 1). Untreated cultures were considered as negative controls. Three independent experiments were carried out by duplicate on each treatment.

\section{Sample preparation}

At the end of treatments, liver slices were washed with ice-cold PBS and transferred to polystyrene tubes containing $500 \mu \mathrm{L}$ of PBS with cocktail protease inhibitors (Roche, IN., USA). After the homogenized samples were centrifuged at $13,000 \mathrm{~g}, 10 \mathrm{~min}, 4^{\circ} \mathrm{C}$, the supernatant was divided into aliquots and stored at $-80^{\circ} \mathrm{C}$ for analysis.

\section{Laboratory analysis}

\section{Reactive oxygen species generation}

ROS generation was assayed with the fluorometric technique 2,4-dichlorofluorescein diacetate (2,4-DCFA) (Wu \& Yotnda 2011). At the end of the exposure, the medium was removed, and cultures were incubated with PBS containing $5 \mu \mathrm{M}$ of 2,4-DCFA (Sigma-Aldrich, St Louis, MO., USA) for $30 \mathrm{~min}$ at $37^{\circ} \mathrm{C}$ on dark conditions. Afterward, fluorescence intensity was measured at $450 \mathrm{~nm}$ emission: 550 excitations using a fluorimeter Versa Fluor TM (Bio-Rad, Hercules, CA, USA).

\section{Determination of Glutathione Peroxidase; glutathione: hydrogen-peroxide oxidoreductase (GPx)}

GPx activity was determined with the Sigma-Aldrich kit (St. Louis, MO., USA). Homogenates were transferred to 96-well polystyrene plates, and kit reagents were added according to the manufacturer's instructions. The change followed enzymatic kinetics in absorbance on a microplate reader Multiskan TM GO (Fisher Scientific, Hampton, NH., USA) at $340 \mathrm{~nm}$.

\section{Total Glutathione Determination}

Total GSH was determined using a 5,5'-dithiobis (2-nitrobenzoic acid) (DTNB) reagent (Sigma-Aldrich, St Louis, MO., USA). Shortly, $25 \mu \mathrm{L}$ from the supernatant were thawed and placed into a 96 well plate, then $175 \mu \mathrm{L}$ of reaction mix (PBS $1 \mathrm{mM}$; EDTA 1mM; DTNB $0.01 \mathrm{M}$ ) was added to the samples and incubated at $37^{\circ} \mathrm{C}$ for $20 \mathrm{~min}$ to obtain a yellow colour formation. Subsequently, the plate scored $450 \mathrm{~nm}$ in a microplate reader. 


\section{Determination of enzymes associated with liver damage}

Alanine transaminase; L-alanine:2-oxoglutarate aminotransferase (ALT), aspartate transaminase; Laspartate:2-oxoglutarate aminotransferase (AST), and y-glutamyl transferase; (5-L-glutamyl)-peptide: amino-acid 5-glutamyltransferase (GGT) bode on the culture medium by using spectrophotometric kits (Spin React, Girona, Spain). The reagents from the diagnostic kits were mixed with the medium culture according to the manufacturer's instructions. The enzymatic kinetics were recorded, considering temperature, wavelength, and time kinetics for each enzyme. L-lactate dehydrogenase; $(S)$-lactate: $N A D^{+}$oxidoreductase (LDH), release was determined by using the cytotoxic kit from Sigma-Aldrich, (St. Louis, MO., USA) the culture media was transferred to 96-well polystyrene plates, and the reagents of the kit were added according to the manufacturer protocol. Then, enzymatic kinetics were measured in a microplate reader.

\section{Zilpaterol and clenbuterol quantification}

Zilpaterol and clenbuterol content in cultures exposed to these beta-agonists were determinate by UPLCMS/MS methodology. Aliquoted samples were defrosted, $100 \mu \mathrm{L}$ and placed into $2 \mathrm{ml}$ polystyrene tubes with $100 \mu \mathrm{L}$ of $\mathrm{NaOH} 5 \mathrm{M}$ and mixed vigorously for 1 minute. Then, $800 \mu \mathrm{L}$ of $\mathrm{ZnOH} 40 \%$ were added and mixed for one minute. After that, the samples were sonicated for $15 \mathrm{~min}$ at $40{ }^{\circ} \mathrm{C}$, allowed to cool, $\mathrm{pH}$ adjusted to 7.0 with $\mathrm{NaOH} 5 \mathrm{M}$, and centrifugate at $13,500 \mathrm{~g}, 10 \mathrm{~min}, 4^{\circ} \mathrm{C}$. The next step consisted of passing the supernatant through cartridges Oasis CMX (Waters, Milford, MA, USA.). The final solution was evaporated on a Turbo Vap LV (Biotage, VA., USA) and reconstituted with ammonium acetate. Reconstituted samples were injected and analysed using ultraperformance liquid chromatography coupled to mass spectrometry (UPLC MS/MS) with a method previously validated (Dolores et al. 2019; Piña-Olmos et al. 2020).

\section{Statistical Analysis}

ANOVA analysed single drug and nanoparticle effects data, and the least significant difference (LSD) test at $95 \%$ was used to identify means statistically differently. Responses of data from combinate drug and nanoparticle were analysed using a factorial data arrangement $2^{2}(2 \times 2)$ to determine the influence of clenbuterol and zilpaterol and their combination with CS-NP or CS/GSH-NP on hepatic enzymes and redox markers. All statistical analyses were performed with STATGRAPHICS Centurion XV Version 15.2.05.

\section{Results}

Individual exposure to clenbuterol, zilpaterol, CS-NP, and CS/GSH-NP in PCLS did not show significantly different effects between treatments on LDH release; enzyme commonly used in the determination of cytotoxicity (Kaja et al. 2017). It has been shown that an increase in LDH release is an indicator of cellular compromise (Kaja et al. 2017; Kumar et al. 2018). There are no significant differences between the treatments and the negative control in the range of concentrations and time used in this work 
(Fig. 2a). The results showed an intracellular amount of zilpaterol and clenbuterol only in the cultures exposed to both $\beta$-adrenergic, and the amount increased according to the concentration used for each one (Fig. 2b). Subsequently, ROS production was determined to know if the $\beta$-agonist drugs and the nanoparticles understudy could modify the amount of these reactive species. The results for individual treatments show ROS generation in a dose-dependent manner on the groups treated with clenbuterol and zilpaterol (Fig. 3a); meanwhile, ROS generation on the groups treated with both types of nanoparticles understudy was lower or similar to the negative control.

ROS and GSH results are consistent with GPx activity; (Fig. 4a) an enzyme whose activity is promoted when there is an increase in peroxide radicals (Ghosh et al. 2019); therefore, an increase in GPx activity is expected when ROS production is elevated on the groups treated with zilpaterol and clenbuterol. In GGT activity, the levels also increased with clenbuterol and zilpaterol (Fig. 4b), which may be related to the need to synthesize GSH to counteract the effects of ROS generated by both drugs in the concentrations used. Similarly, as GPx and GGT, the results of the individual treatments in the determination of AST and ALT (Fig. 5a-b) showed an increase in the high concentrations of clenbuterol and zilpaterol studied.

Regarding the combined effects, treatments in which both drugs were combined with CS-NP showed significant changes in ROS production; meanwhile, ROS levels increasing in the case of clenbuterol (Fig. 6a), the combination of CS-NP with zilpaterol demonstrate an opposite effect (Fig. 6b). When CS/GSH-NPs were used, the induction of reactive species was not significantly modified. Therefore, these results suggest that exogen GSH delivered by nanoparticles in the cell could have been used to modulate oxidation-reduction stress or some events associated with liver biotransformation when the tissue was exposed to both drugs.

On the other hand, regardless of the type of nanoparticles used, promoted the response of GPx activity estimated in the combined groups with clenbuterol regardless of the type of nanoparticles used (Fig. 7ab). Zilpaterol group combined with CS-NP also increased the response of GPx (Fig. 7c). However, the combined treatment of zilpaterol with CS/GSH-NP, was observed to reduce in GPx activity (Fig. 7d); that could be related to an increase in the amount of GSH (Fig. 8b). These findings suggest that GSH can be incorporated into cells through nanoparticles, and the response of exposed PCLS to both agonists $\beta$ adrenergic and exogenous GSH is different for each one. Also, CS/GSH-NP significantly reduces the GGT response in the PCLS treated with clenbuterol (Fig. 9b), while this effect in the combined treatments with zilpaterol was not significantly modified.

A significant response reduced ALT release in the groups exposed to clenbuterol combined with CS-NP (Fig. 10a) and CS/GSH-NP (Fig. 10b). This reduction also occurred in PCLS exposed to zilpaterol and CSNP (Fig. 10c), surprisingly occurs the opposite in cultures exposed to zilpaterol with CS/GSH-NP (Fig. 10d). These results suggest a differential sensitivity of bovine liver tissue when is exposed to both drugs and the nanoparticles under study. In AST, the response also reduced on both agonists $\beta$-adrenergic combined with CS-NP (Fig. 11a-b); whereas CS/GSH-NP does not modify the AST response during the combined treatment with zilpaterol or clenbuterol. 


\section{Discussion}

Previous works demonstrate that clenbuterol metabolism promotes the formation of reactive oxygen and nitrogen metabolites or species that react with biomolecules to form covalent adducts (Brambilla et al. 2007; Pietraforte et al. 2008). Regarding zilpaterol, there are no data about reactive metabolites. However, some studies have shown an increase in the mortality of cattle (Loneragan et al. 2014), increase in nonesterified fatty acids (Parr et al. 2014), increased ovarian weight, cyst and leiomyoma formation in rats (Arcella et al. 2016), as well as toxic effects after administration in horses like the presence of tremors, increased of LDH, creatinine kinase, and AST biomarkers of liver and muscle damage (Hepworth-Warren \& Alcott 2014; Wagner et al. 2008).

A way to protect the cells and maintain redox balance in the organisms is by transforming or conjugating the reactive species into less harmful ones to avoid cell damage. In that sense, intracellular glutathione plays a fundamental role in antioxidant defence. Besides, it participates in liver metabolism, promoting xenobiotic detoxification reactions through oxidation to GSSG and/or conjugation with reactive or intermediate metabolites (Geenen et al. 2013; Stanley 2016; Zamek-Gliszczynski et al. 2006). Bearing in mind, viable cultures exposed to clenbuterol and zilpaterol could produce ROS and because the oxidereduction system depends mainly on the modulation exerted by GSH we used nanoparticles with GSH and our results showed a significant difference in GSH levels after exposure to clenbuterol $(25 \mathrm{ng} / \mathrm{ml})$ compared to the negative control and the low concentration of CS/GSH-NP. Because high amounts of reactive oxygen species make the cells respond by producing antioxidant enzymes such as superoxide dismutase, catalase, glutathione peroxidase, and antioxidant molecules like glutathione (Ighodaro \& Akinloye 2018; Wu \& Yotnda 2011). In this work GPx and GGT were elevated. GGT regulates the cysteine levels that contribute to the synthesis of GSH (Hanigan 2014). A relationship has been observed in increasing of this enzyme in various types of tumours with high amounts of intracellular GSH (Hanigan 2014) and it is considered a biomarker of hepatocellular damage (Tong et al. 2020). Other functions of GGT are to catalyse the transfer of gamma-glutamyl groups from different cellular sources (Whitfield 2001), such as intracellular GSH, which could be incorporated through nanoparticles. Therefore, this enzyme also plays a fundamental role in the regulation of GSH synthesis. Since the amount of GSH is optimal in the cell, GGT activity is decreased or moderate, whereby the GSH tripeptide as a potent inhibitor of that enzyme (Hoffmann \& Solter 2008; Phelps \& Mayer 2012); moreover, GGT catalyses the transfer of gamma-glutamyl groups of GSH-conjugated metabolites during xenobiotic detoxification (Hanigan 2014; Hoffmann \& Solter 2008).

ALT and AST are common biomarkers of liver damage associated with viral and bacterial diseases, substance abuse, and inflammation (Anadón et al. 2019; Aulbach \& Amuzie 2017; Hoffmann \& Solter, 2008). Greater ALT use than AST has been documented since its values have a more significant correlation and specificity with different liver risk events. AST is also present in other organs and tissues, hence its lower specificity with liver damage. 
These enzymes are also associated with liver damage (Meunier \& Larrey 2019); their increase could be related to several physiological events such as hepatitis, diabetes mellitus (Gan et al. 2020), hyperthyroidism (Sato et al. 2010), or toxicity induced by drugs such as acetaminophen (Dear \& Bateman

2019), including some types of nanoparticles (Rasool et al. 2020). The administration of drugs can cause liver damage through two ways in which liver cells are affected by their administration; one is dosedependent, the other can be variable and has been related to hypersensitivity events or other factors not described yet and in most cases is independent of the dose (Meunier \& Larrey 2019; Weiler et al. 2015). Therefore, it is crucial to evaluate and develop other specific biomarkers that give more information about the toxic effects observed in risk population exposed to this kind of agonist $\beta$-adrenergic directly or indirectly through food consumption from animal origin (Meunier \& Larrey 2019; Weiler et al. 2015).

In the specific case of the agonists $\beta$-adrenergic studied in this study; there are no well-established adverse effects at the cellular and molecular level that cause the reactive or intermediate metabolites formed during phase I and II reactions; these can bind to proteins, DNA, among other biomolecules affecting cellular signals that compromise the oxide-reduction state and cellular homeostasis (Curbo et al. 2013). 35432. Hence the importance of studies at the cellular and molecular level of drugs such as clenbuterol and zilpaterol, which are used in animals for human consumption, puts health at significant risk when used clandestinely or the therapeutic doses and retirement time is exceeded. On the other hand, having delivery systems of antioxidant molecules such as the nanoparticles studied in this work allows the incorporation of glutathione in cells susceptible to oxidative damage caused by xenobiotic and allow us to go forward in the study of the response to these agonists in glutathione intracellular presence.

The results obtained in this work suggest that the hepatic biochemical mechanisms on bovine PCLS dependent on GPx, GGT, ALT, and AST can be modified by delivering antioxidant molecules such as glutathione into the cells by using nanoparticles. Therefore, the impact of those compounds on redox markers and transaminases release could be associated with the formation of reactive species; from here, the importance of extending the cellular and molecular evaluation of drugs like clenbuterol and zilpaterol, which are employed in animal production to contribute to the knowledge of the toxicological features at the cellular level of both agonists $\beta$-adrenergic.

\section{Declarations}

\section{Acknowledgements}

The authors thank Jesús Américo Hernández Góngora and Luis Alberto Parra Oaxaca for providing bovine livers from cattle destined for human consumption

\section{Funding}

This work was supported by the National University Autonomous of México DGAPA-PAPIIT IT201620 and PAPIIT IN214321. Sofia Piña Olmos received by the National Council of Science and Technology (CONACYT) doctoral fellowship 289464. 


\section{Compliance with ethical standards}

This study used bovine liver samples obtained from animals slaughtered in the municipal slaughterhouse of Zumpango, Mexico State, Mexico. The animals were destinated for human consumption. All the procedures used were according to the local care legislation NOM-033-SAG/ZOO-2014 and the suggested methods to slaughter domestic and wild animals (Diario Oficial de la Federación 2015).

\section{Author contributions}

Patricia Ramírez Noguera, Roberto Díaz Torres and Sofía Piña Olmos contributed to the study conception and design. Material preparation, data collection and analyses were performed by Sofía Piña Olmos, Mariana Dolores-Hernández, Raquel López Arellano, Roberto Díaz Torres and Efrén Ramírez Bribiesca. The first draft of the manuscript was written by Sofía Piña Olmos and Patricia Ramírez Noguera and all authors commented on previous versions of the manuscript. All authors read and approved the final manuscript.

Conflicts of interest The authors declare no conflict of interest.

Consent to participate Not applicable.

Consent for publication All authors give consent for publication.

Code availability Not applicable.

\section{References}

1. Alonen A, Gartman M, Aitio O, Finel M, Yli-Kauhaluoma J, Kostiainen R (2009) Synthesis, structure characterization, and enzyme screening of clenbuterol glucuronides. Eur J Pharm Sci 37:581-587

2. Brambilla G, di Bez S, Pietraforte D, Minetti M, Campanella L, Loizzo A (2007) Ex vivo formation of gastric metabolites of clenbuterol: Preliminary characterisation of their chemical structure. Anal Chim Acta 586:426-431

3. Centner TJ, Alvey JC, Stelzleni AM (2014) Beta-agonists in livestock feed: Status, health concerns, and international trade. J Anim Sci 92:4234-4240

4. Choi CB, Jung KK, Chung KY, Yang BS, Chin KB, Suh SW, Oh DH, Jeon MS, Baek KH, Lee SO et al (2013) Administration of zilpaterol hydrochloride alters feedlot performance, carcass characteristics, muscle, and fat profiling in finishing Hanwoo steers. Livest Sci 157:435-441

5. Cônsolo NRB, Ferrari VB, Mesquita LG, Goulart RS, Silva LFP (2016) Zilpaterol hydrochloride improves beef yield, changes palatability traits, and increases calpain-calpastatin gene expression in Nellore heifers. Meat Sci 121:375-381

6. Diario Oficial de la Federación (2015) Norma Oficial Mexicana: NOM-033-SAG/ZOO-2014. Métodos para dar muerte a los animales domésticos y silvestres (Methods to slaughter domestic and wild animals) 
7. Dolores HM, Villaseñor A, Piña OS, Mercado Márquez C, Bejarano BV, Bonaparte MEG, López-Arellano $\mathrm{R}$ (2019) Evaluation of R- (-) and S- (+) Clenbuterol enantiomers during a doping cycle or continuous ingestion of contaminated meat using chiral liquid chromatography by LC-TQ-MS. Drug Test Anal $11: 1238-1247$

8. Domínguez-Romero JC, García-Reyes JF, Martínez-Romero R, Martínez-Lara E, Del Moral-Leal ML, Molina-Díaz A (2013) Detection of main urinary metabolites of $\beta 2$-agonists clenbuterol, salbutamol and terbutaline by liquid chromatography high resolution mass spectrometry. J Chromatogr B Anal Technol Biomed Life Sci 923-924:128-135

9. FAO and WHO Expert Committee on Food Additives (2014) Evaluation of certain veterinary drug residues in food. Toxicol Eval Certain Vet drug residues food. Prep by seventy-eighth Meet Jt FAO and WHO Expert Comm Food Addit (JECFA)Series 69

10. FAO and WHO Expert Committee on Food Additives (2016) Evaluation of certain veterinary drug residues in food. Eighty-first report of the Joint FAO and WHO Expert Committee on Food Additives. World Heal Organ Tech Rep Ser 997:1-110

11. FAO and WHO Expert Committee on Food Additives (2018) Evaluation of certain veterinary drug residues in food: eighty-fifth report of the Joint FAO andWHO Expert Committee on Food Additives. World Health Organ Tech Rep Ser, Geneva, p 1008

12. Granitzny A, Knebel J, Schaudien D, Braun A, Steinberg P, Dasenbrock C, Hansen T (2017) Maintenance of high-quality rat precision-cut liver slices during culture to study hepatotoxic responses: Acetaminophen as a model compound. Toxicol Vitr 42:200-213

13. He L, He T, Farrar S, Ji L, Liu T, Ma X (2017) Antioxidants Maintain Cellular Redox Homeostasis by Elimination of Reactive Oxygen Species. Cell Physiol Biochem 44:532-553

14. Hejjaji EMA, Smith AM, Morris GA (2018) Evaluation of the mucoadhesive properties of chitosan nanoparticles prepared using different chitosan to tripolyphosphate (CS: TPP) ratios. Int J Biol Macromol 120:1610-1617

15. Kafedjiiski K, Föger F, Werle M, Bernkop-Schnürch A (2005) Synthesis and in vitro evaluation of a novel chitosan-glutathione conjugate. Pharm Res 22:1480-1488

16. Koo SH, Lee JS, Kim GH, Lee HG (2011) Preparation, characteristics, and stability of glutathioneloaded nanoparticles. J Agric Food Chem 59:11264-11269

17. Kumar R, Sinha VR (2013) Thiomer: A potential carrier for therapeutic delivery. React Funct Polym 73:1156-1166

18. Lu SC (2009) Regulation of glutathione synthesis. Mol Aspects Med 30:42-59

19. Lu SC (2013) Glutathione synthesis. Biochim Biophys Acta - Gen Subj 1830:3143-3153

20. Lu SC (2020) Dysregulation of glutathione synthesis in liver disease. Liver Res 4:64-73

21. Masubuchi N, Nishiya T, Imaoka M, Mizumaki K, Okazaki O (2016) Promising toxicological biomarkers for the diagnosis of liver injury types: Bile acid metabolic profiles and oxidative stress marker as screening tools in drug development. Chem Biol Interact 255:74-82 
22. Milford AB, Le Mouël C, Bodirsky BL, Rolinski S (2019) Drivers of meat consumption. Appetite. 141(January)

23. Olinga P, Schuppan D (2013) Precision-cut liver slices: A tool to model the liver ex vivo. J Hepatol 58:1252-1253

24. Patlevič $P$, Vašková J, Švorc $P$, Vaško L, Švorc $P(2016)$ Reactive oxygen species and antioxidant defence in human gastrointestinal diseases. Integr Med Res 5:250-258

25. Piña-Olmos S, Díaz-Torres R, Elbakrawy E, Hughes L, McKenna J, Hill MA, Kadhim M, Ramírez-, Noguera P, Bolanos-Garcia VM (2019) Combinatorial use of chitosan nanoparticles, reversine, and ionising radiation on breast cancer cells associated with mitosis deregulation. Biomolecules 9:1-12

26. Piña-Olmos S, Dolores-Hernández M, Villaseñor A, Díaz-Torres R, Ramírez Bribiesca E, López-Arellano R, Ramírez-Noguera P (2020) Extracellular and intracellular zilpaterol and clenbuterol quantification in Hep G2 liver cells by UPLC-PDA and UPLC-MS/MS. J Pharm Biomed Anal.195

27. Ruoß M, Vosough M, Königsrainer A, Nadalin S, Wagner S, Sajadian S, Huber D, Heydari Z, Ehnert S, Hengstler JG, Nussler AK (2020) Towards improved hepatocyte cultures: Progress and limitations. Food Chem Toxicol. 138

28. Soldatow VY, Lecluyse EL, Griffith LG, Rusyn I (2013) In vitro models for liver toxicity testing. Toxicol Res 2:23-39

29. Van der Lubben IM, Verhoef JC, Borchard G, Junginger HE (2001) Chitosan and its derivatives in mucosal drug and vaccine delivery. Eur J Pharm Sci 14:201-207

30. Wu D, Yotnda P (2011) Production and detection of reactive oxygen species (ROS) in cancers. J Vis Exp 57:2-5

31. Zalko D, Debrauwer L, Bories G, Tulliez J (1997) Evidence for a new and major metabolic pathway of clenbuterol involving in vivo formation of an N-hydroxy arylamine. Chem Res Toxicol 10:197-204

\section{Figures}




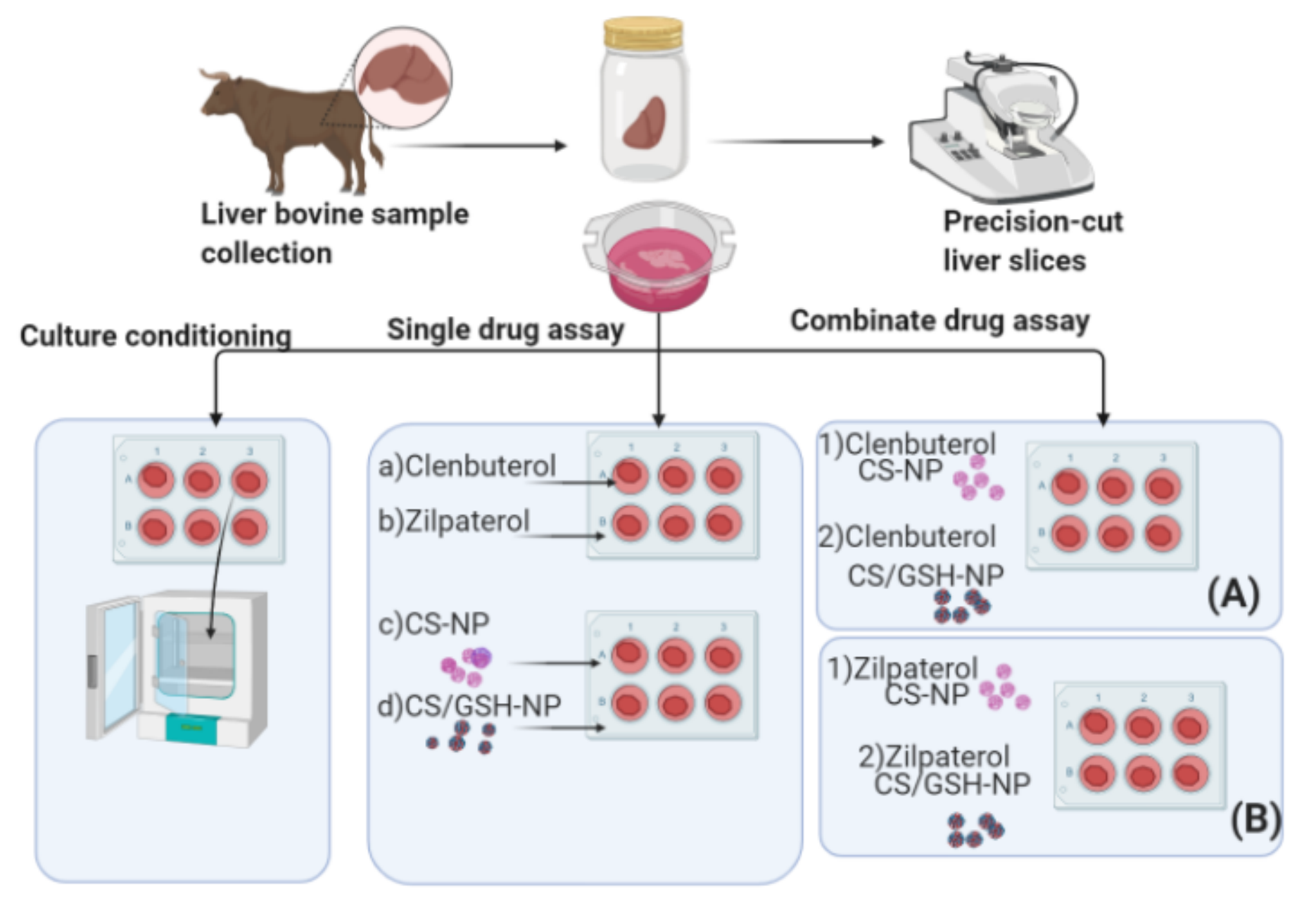

\section{Figure 1}

Single drug and nanoparticles exposure Single treatments were carried out on PCLS treated with two concentrations of zilpaterol ( 10 and $25 \mathrm{ng} / \mathrm{mL}$ ), clenbuterol (10 and $25 \mathrm{ng} / \mathrm{mL}$ ), CS-NP (10 and 25 $\mu \mathrm{g} / \mathrm{mL}$ ), and CS/GSH-NP (10 and $25 \mu \mathrm{M} \mathrm{GSH}$ ) and left under culture conditions for 6 hours (Fig. 1), For good measure, cultures were harvested and stored at $-80^{\circ} \mathrm{C}$. An unthreaded group culture was considered as the negative control.
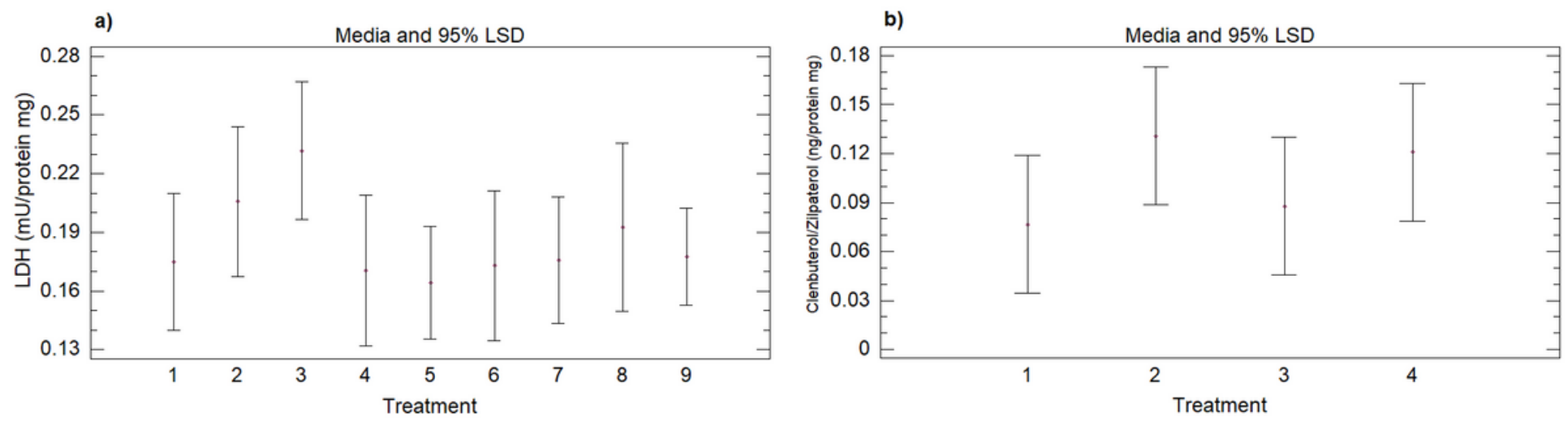

Figure 2 
Individual exposure to clenbuterol, zilpaterol, CS-NP, and CS/GSH-NP in PCLS did not show significantly different effects between treatments on LDH release; enzyme commonly used in the determination of cytotoxicity (Kaja et al. 2017). It has been shown that an increase in LDH release is an indicator of cellular compromise (Kaja et al. 2017; Kumar et al. 2018). There are no significant differences between the treatments and the negative control in the range of concentrations and time used in this work (Fig. 2a). The results showed an intracellular amount of zilpaterol and clenbuterol only in the cultures exposed to both $\beta$-adrenergic, and the amount increased according to the concentration used for each one (Fig. $2 b)$.
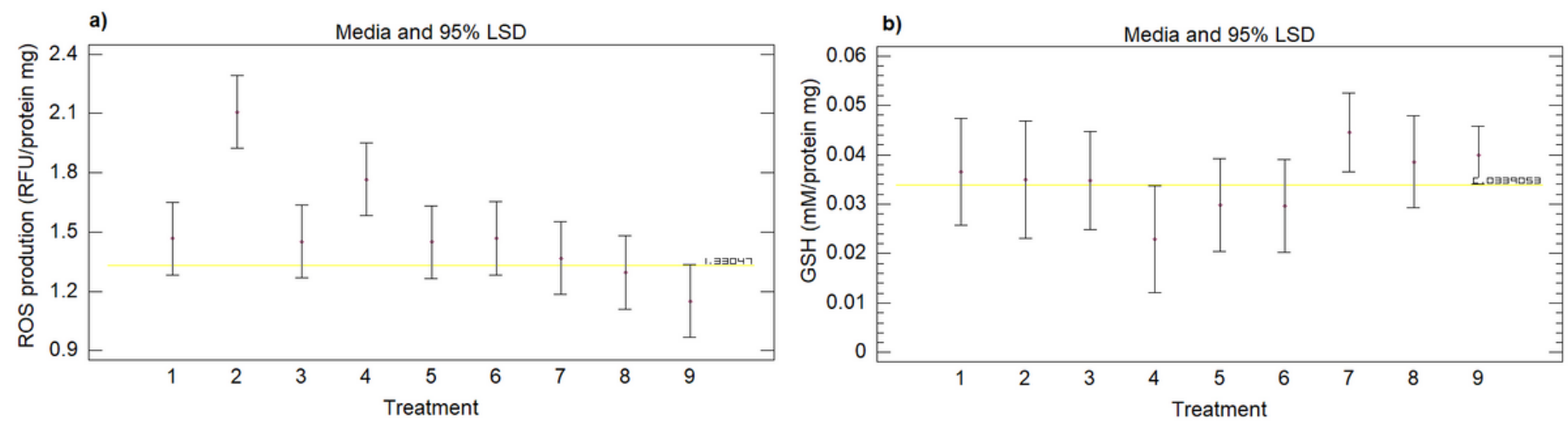

\section{Figure 3}

Subsequently, ROS production was determined to know if the $\beta$-agonist drugs and the nanoparticles understudy could modify the amount of these reactive species. The results for individual treatments show ROS generation in a dose-dependent manner on the groups treated with clenbuterol and zilpaterol (Fig. 3a); meanwhile, ROS generation on the groups treated with both types of nanoparticles understudy was lower or similar to the negative control.
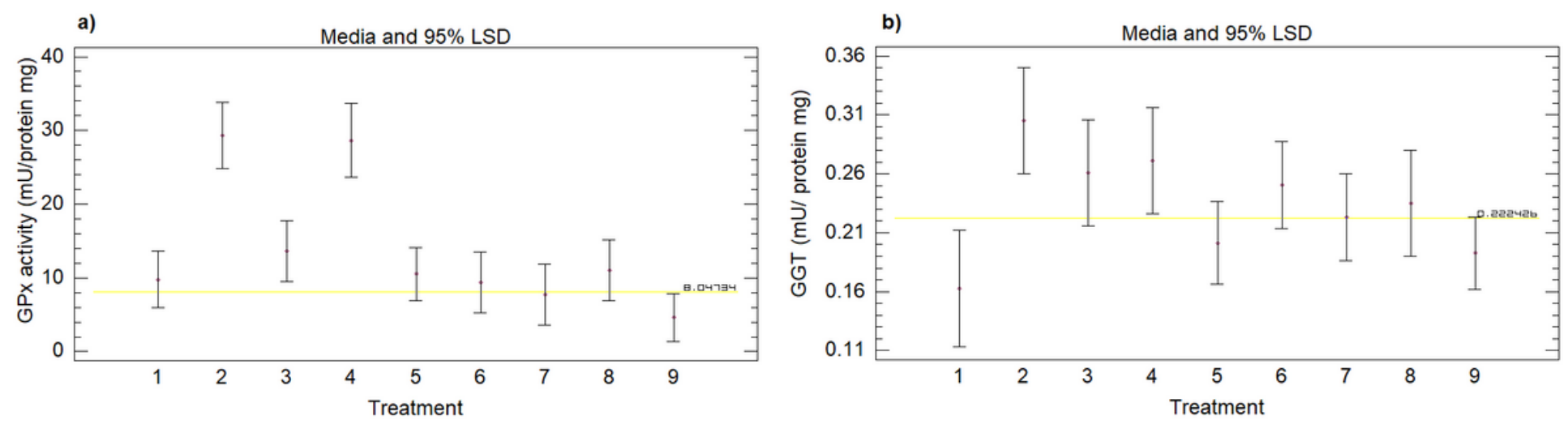

\section{Figure 4}

ROS and GSH results are consistent with GPx activity; (Fig. 4a) an enzyme whose activity is promoted when there is an increase in peroxide radicals (Ghosh et al. 2019); therefore, an increase in GPx activity is expected when ROS production is elevated on the groups treated with zilpaterol and clenbuterol. In GGT activity, the levels also increased with clenbuterol and zilpaterol (Fig. 4b), 

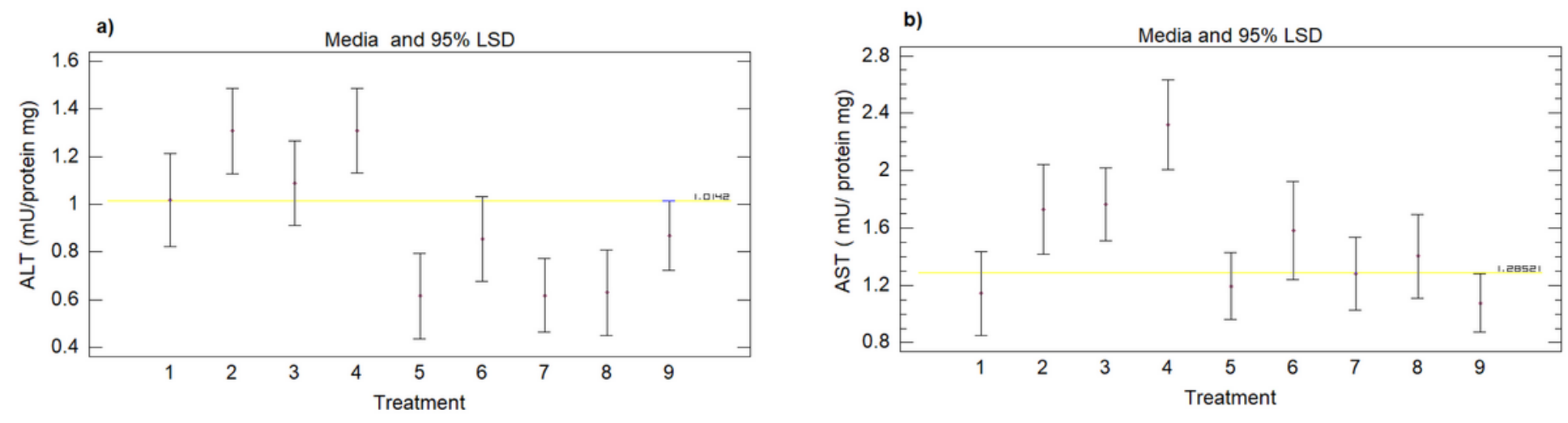

\section{Figure 5}

synthesize GSH to counteract the effects of ROS generated by both drugs in the concentrations used. Similarly, as GPx and GGT, the results of the individual treatments in the determination of AST and ALT (Fig. 5 a-b) showed an increase in the high concentrations of clenbuterol and zilpaterol studied.
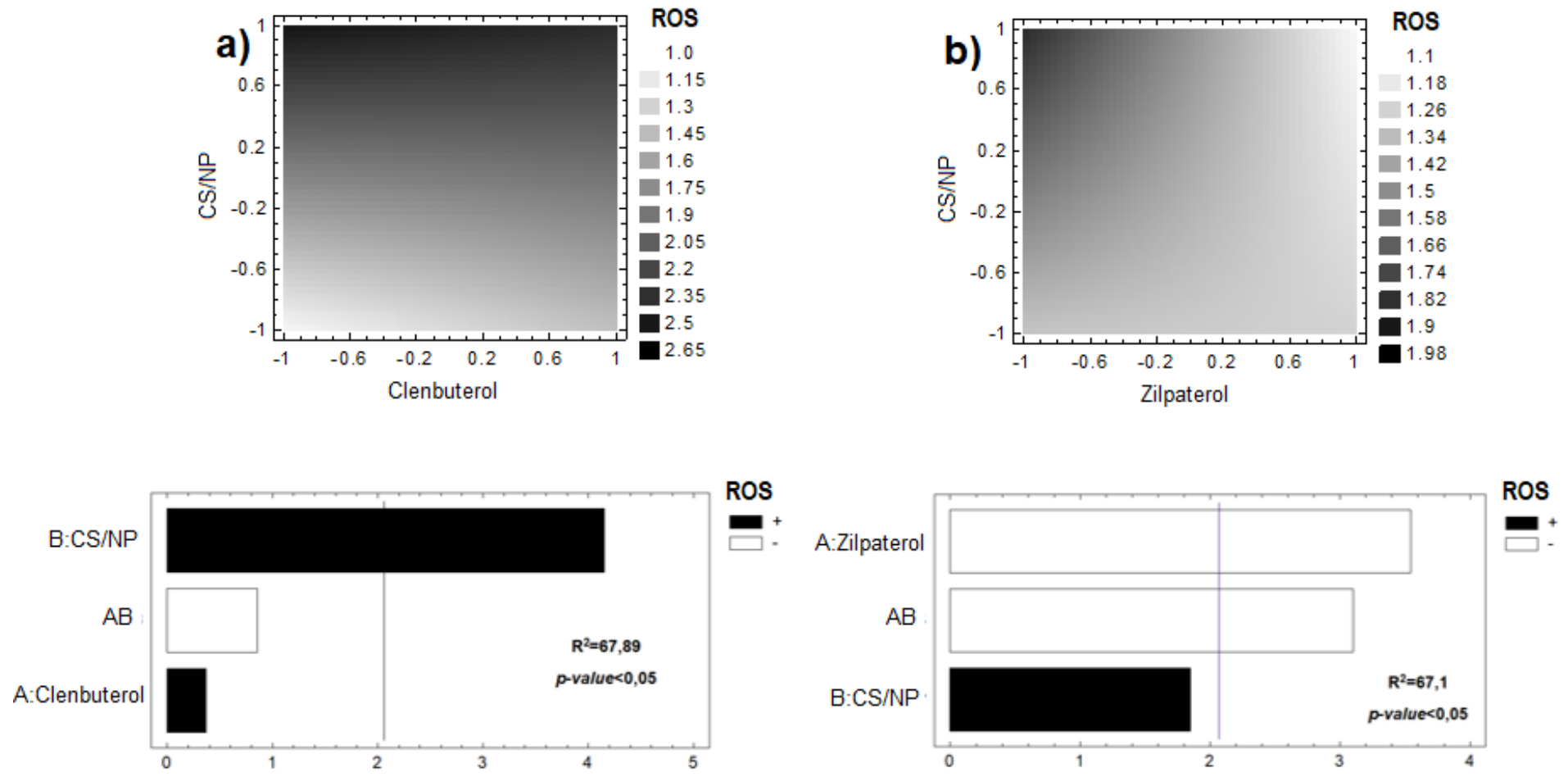

\section{Figure 6}

Regarding the combined effects, treatments in which both drugs were combined with CS-NP showed significant changes in ROS production; meanwhile, ROS levels increasing in the case of clenbuterol (Fig. 6 a), the combination of CS-NP with zilpaterol demonstrate an opposite effect (Fig. 6 b). When CS/GSHNPs were used, the induction of reactive species was not significantly modified. Therefore, these results suggest that exogen GSH delivered by nanoparticles in the cell could have been used to modulate oxidation-reduction stress or some events associated with liver biotransformation when the tissue was exposed to both drugs. 

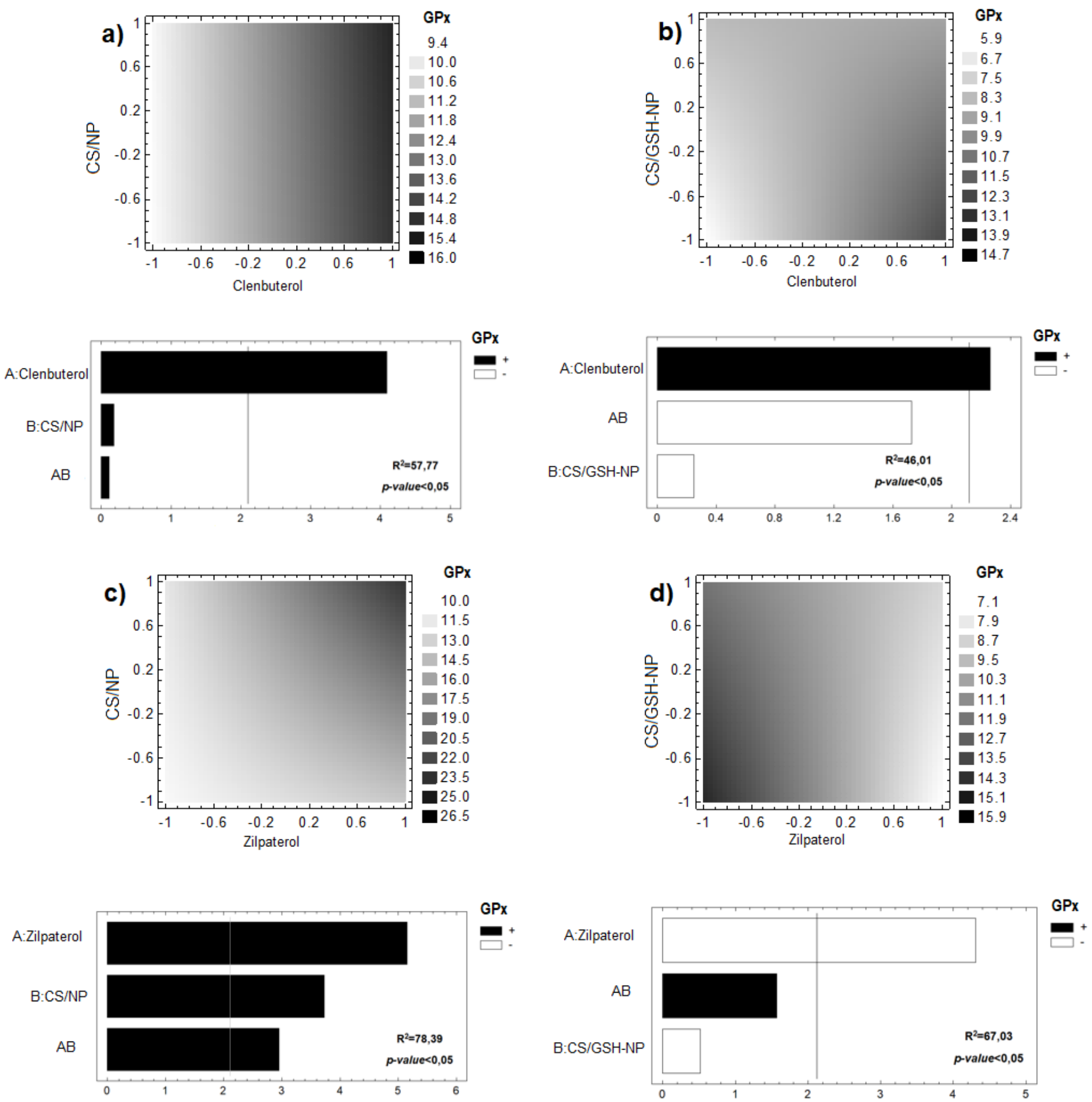

\section{Figure 7}

On the other hand, regardless of the type of nanoparticles used, promoted the response of GPx activity estimated in the combined groups with clenbuterol regardless of the type of nanoparticles used (Fig. 7 ab). Zilpaterol group combined with CS-NP also increased the response of GPx (Fig. 7c). However, the combined treatment of zilpaterol with CS/GSH-NP, was observed to reduce in GPx activity (Fig. 7d); that could be related to an increase in the amount of GSH (Fig. 8b). 

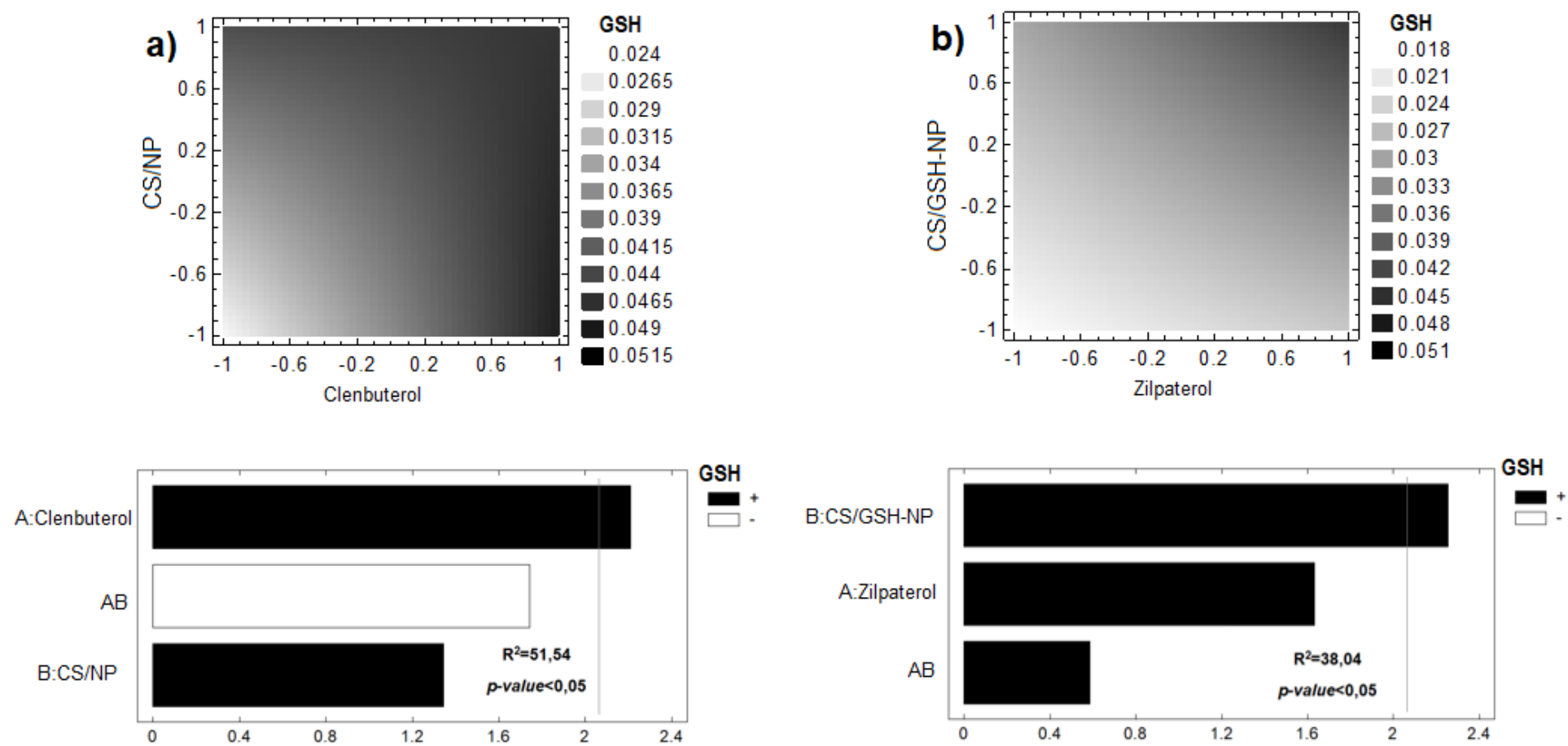

\section{Figure 8}

However, the combined treatment of zilpaterol with CS/GSH-NP, was observed to reduce in GPx activity (Fig. 7d); that could be related to an increase in the amount of GSH (Fig. 8b).
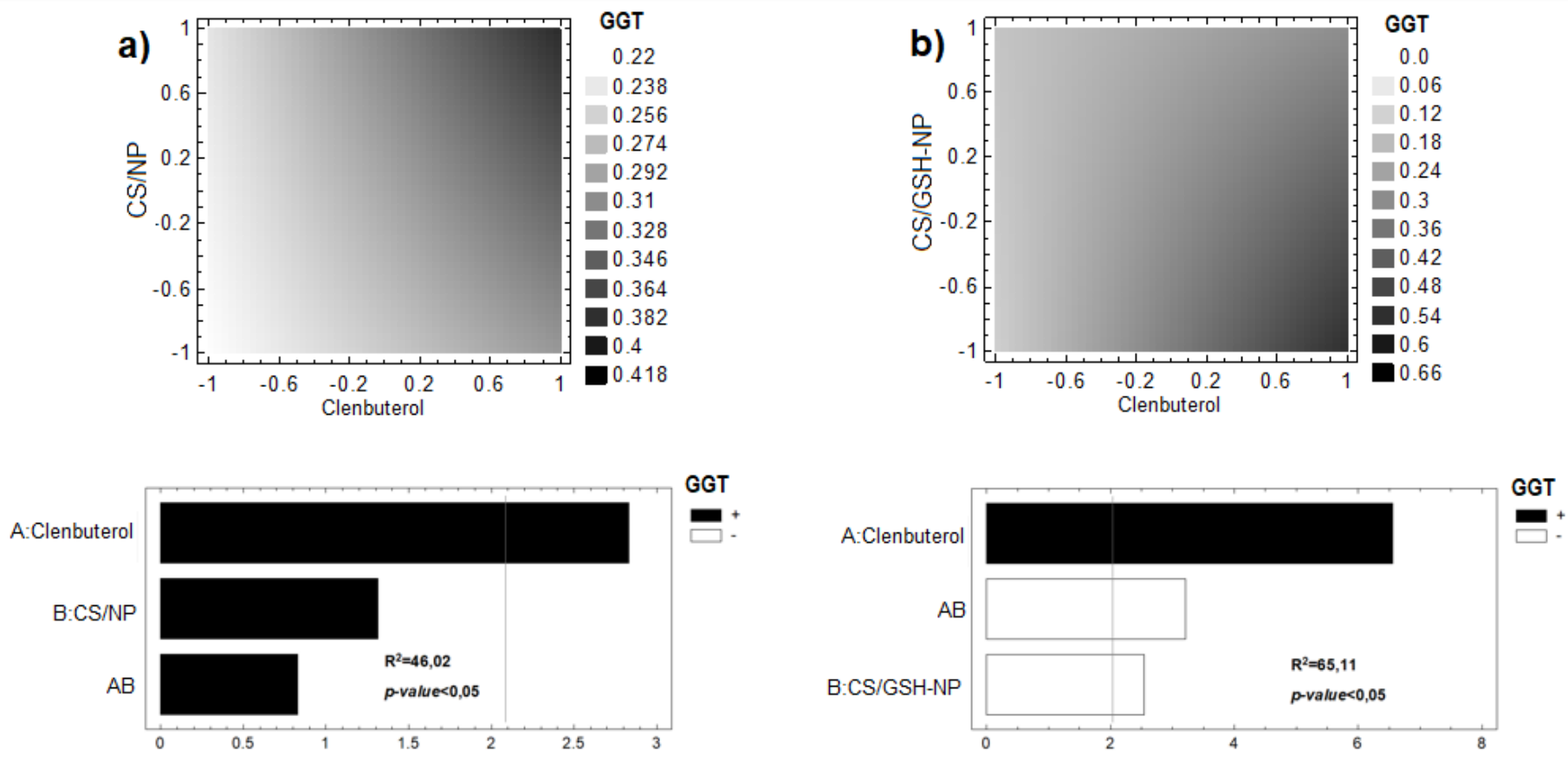

\section{Figure 9}

These findings suggest that GSH can be incorporated into cells through nanoparticles, and the response of exposed PCLS to both agonists $\beta$-adrenergic and exogenous GSH is different for each one. Also, 
CS/GSH-NP significantly reduces the GGT response in the PCLS treated with clenbuterol (Fig. 9b), while this effect in the combined treatments with zilpaterol was not significantly modified.
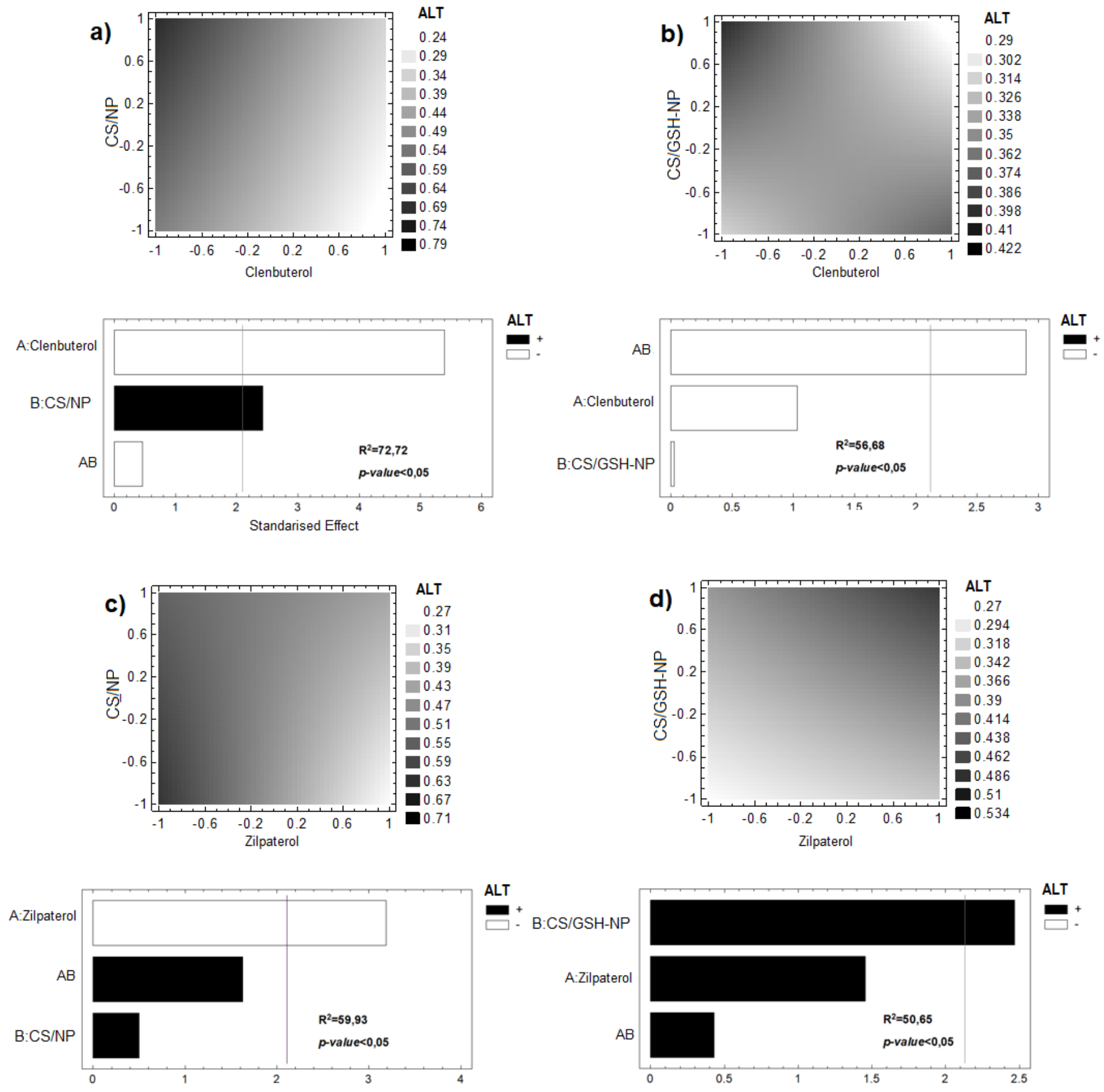

\section{Figure 10}

A significant response reduced ALT release in the groups exposed to clenbuterol combined with CS-NP (Fig. 10 a) and CS/GSH-NP (Fig. 10 b). This reduction also occurred in PCLS exposed to zilpaterol and 
CS-NP (Fig. 10 c), surprisingly occurs the opposite in cultures exposed to zilpaterol with CS/GSH-NP (Fig. $10 \mathrm{~d})$.
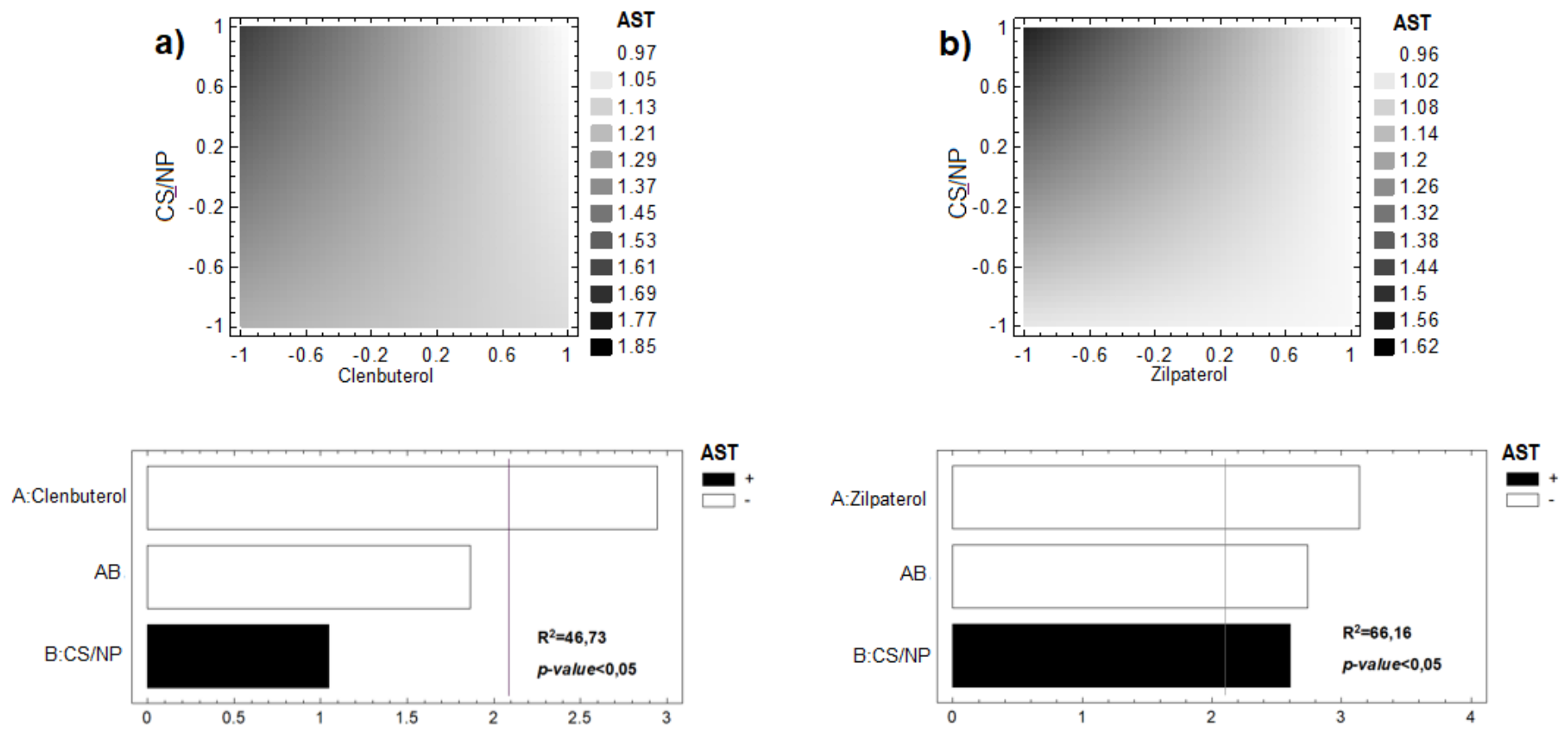

\section{Figure 11}

These results suggest a differential sensitivity of bovine liver tissue when is exposed to both drugs and the nanoparticles under study. In AST, the response also reduced on both agonists $\beta$-adrenergic combined with CS-NP (Fig. 11 a-b); whereas CS/GSH-NP does not modify the AST response during the combined treatment with zilpaterol or clenbuterol. 